\title{
Conjugate Gradient Method to Solve Fluid Structure Interaction Problem
}

\author{
Mamadou Diop', Ibrahima Mbaye ${ }^{2}$ \\ ${ }^{1}$ Thies University, Thies, Senegal \\ ${ }^{2}$ Department of Mathematics and Computer Science, Thies University, Thies, Senegal \\ Email: mamadou.diop@univ-thies.sn,i.mbaye@univ-thies.sn
}

How to cite this paper: Diop, $M$. and Mbaye, I. (2017) Conjugate Gradient Method to Solve Fluid Structure Interaction Problem. Applied Mathematics, 8, 444-452. https://doi.org/10.4236/am.2017.84036

Received: February 18, 2017

Accepted: April 21, 2017

Published: April 24, 2017

Copyright (c) 2017 by authors and Scientific Research Publishing Inc. This work is licensed under the Creative Commons Attribution International License (CC BY 4.0).

http://creativecommons.org/licenses/by/4.0/

\section{(c) (i) Open Access}

\begin{abstract}
In this paper, we propose a method to solve coupled problem. Our computational method is mainly based on conjugate gradient algorithm. We use finite difference method for the structure and finite element method for the fluid. Conjugate gradient method gives suitable numerical results according to some papers.
\end{abstract}

\section{Keywords}

Fluid-Structure Interaction, Beam, Stokes, Finite Element, Finite Difference Method, Conjugate Gradient Method

\section{Introduction}

Problem involving fluid structure interaction occurs in a wide vatiety of engineering problems and therefore has attracted the interest of many investigations from different engineering disciplines. As a result, much efforts has gone into the development of general computational method for fluid structure systems [1] [2] [3] [4] [5] [6].

Amongst the computational methods for fluid structure interaction problem, we cite the fixed point method, the Newton method, the Quasi-Newton method, the fictitious domain method. In this work we present a method based on the conjugate gradient algorithm. In effect, the fluid interaction problems occur in biomedical fluids areas for example blood flow interaction with elastic veins. Thus, this paper aims at showing that, we can combine the finite difference method, the finite element method and the conjugate gradient method to solve fluid structure interaction problem. On the one hand, we use finite difference method to approximate the structure model in order to have a linear systems, On the other hand, we solve the stokes equation by the finite element method. Moreover, 
conjugate gradient method will be intruduced to compute the displacement of the structure. Thus, the velocity $v$ and the pressure $p$ of the fluid are done in the deformed domain. In addition, the fluid represented by the blood is modelled by two dimensional Stokes equation for steady flow and the structure represented by the body vessel is modelled by the one dimensional beam equation.

\section{Position of Problem}

\subsection{Domain Fluid}

The fluid domain noted $\Omega_{F}^{u} \subset \mathbb{R}^{2}$ is represented in the Figure 1 .

Where, the border $\partial \Omega_{F}^{u}=\Sigma_{1} \cup \Sigma_{2} \cup \Sigma_{3} \cup \Gamma_{2}$.

- $\Gamma_{2}$ is the interface between the fluid and the elastic structure

- $\Sigma_{1}$ is The inflow

- $\Sigma_{2}$ is a rigid border

- $\Sigma_{3}$ is the outflow

- $L$ is the domain length

- $H$ is the domain height

- $u$ is the displacement of the structure

\subsection{Fluid Properties}

The fluid is considered to be Newtonian, incompressible and its state is described by the velocity $v=\left(v_{1}, v_{2}\right)$ and the pressure $p$. The balance equations are

$$
\begin{gathered}
-\mu \Delta v+\nabla p=f^{F}, \text { in } \Omega_{F}^{u} \\
\nabla \cdot v=0, \text { in } \Omega_{F}^{u} \\
v=G, \text { on } \Sigma_{1} \cup \Sigma_{2} \\
v=0, \text { on } \Gamma_{2} \\
-p I_{d} n+\frac{\partial v}{\partial n}=0, \text { on } \Sigma_{3}
\end{gathered}
$$

- $\mu$ : the fluid viscosity

- $I_{d}$ the identity matrix

- $f^{F}=\left(f_{1}^{F}, f_{2}^{F}\right)$ the volume force of the fluid

- $n$ is a unit normal vector

- $G=\left(G_{1}, G_{2}\right)$ the velocity profil in $\Sigma_{1}$

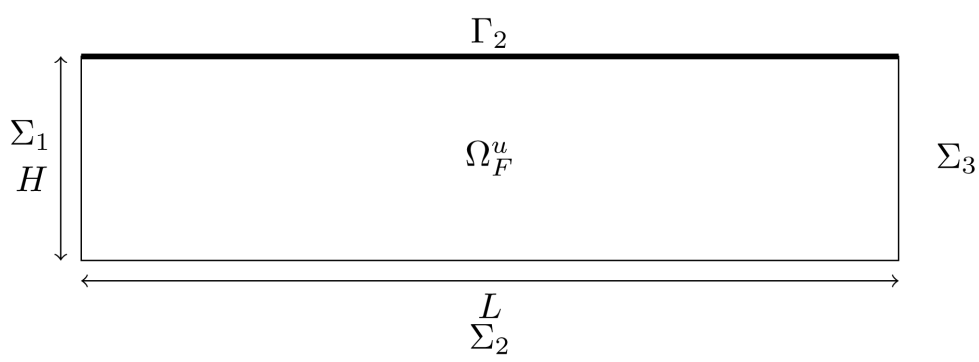

Figure 1. Reference domain. 


\subsection{Structure Properties}

The structure is assumed by elastic beam. We note $u:[0, L] \rightarrow \mathbb{R}$ the displacement of the structure, it is modelled by the beam equation

$$
D u^{(4)}(x)=p(x, H+u(x)), \forall x \in[0, L],
$$

with the boundary conditions,

$$
\begin{aligned}
u(0) & =u(L)=0 \\
u^{\prime}(0) & =u^{\prime}(L)=0
\end{aligned}
$$

where,

- $D=\frac{E \times h^{3}}{12\left(1-v^{2}\right)}$

- $E$ is the Young modulus

- $h$ elastic structure thickness

- $v$ the Poisson's coefficient

Remark: In Equation (6) we assume that only the pressure force is acting on the interface and also $u$ is the transversal displacement [3].

\section{Coupled Problem}

The coupled problem is to find $(u, v, p)$ such that:

$$
\left\{\begin{array}{l}
D u^{(4)}(x)=p(x, H+u(x)) \forall x \in[0, L] \\
u(0)=u(L)=0 \\
u^{\prime}(0)=u^{\prime}(L)=0 \\
-\mu \Delta v+\nabla p=f^{F}, \text { in } \Omega_{F}^{u} \\
\nabla \cdot v=0, \text { in } \Omega_{F}^{u} \\
v=G, \text { on } \Sigma_{1} \cup \Sigma_{2} \\
v=0, \text { on } \Gamma_{2} \\
-p I_{d} n+\frac{\partial v}{\partial n}=0, \text { on } \Sigma_{3}
\end{array}\right.
$$

In order to solve this coupled problem, we transform its continuous problem into a discreet problem by using finite difference method and finite element method.

\subsection{Approximation by Taylor Development}

Assumption: We consider $u$ as a small displacement.

Thus, the Taylor formula gives

$$
p(x, H+u(x)) \approx p(x, H)+u(x) \frac{\partial p(x, H)}{\partial y},
$$

the Equation (6) becomes:

$$
D u^{(4)}(x)-u(x) \frac{\partial p(x, H)}{\partial y}=p(x, H),
$$

we pose $\alpha(x)=-\frac{\partial p(x, H)}{\partial y}$, finally we have, 


$$
D u^{(4)}(x)+\alpha(x) u(x)=p(x, H) .
$$

To discretize the Equation (11), we introduce a space step $\Delta x=\frac{L}{N+1}$. We denote by $u_{i}$ the value of the discrete solution at $x_{i}=i \times \Delta x$ for $i \in\{0,1, \cdots, N+1\}$. We must also discretize The boundary conditions . A centred formula gives

$$
u_{1}=u_{-1} \text { et } u_{N+2}=u_{N}
$$

and the boundary conditions $u(0)=u(L)=0$ become

$$
u_{0}=u_{N+1}=0
$$

we rewrite the Equation (11) in the discreet form

$$
D \frac{u_{i-2}-4 u_{i-1}+6 u_{i}-4 u_{i+1}+u_{i+2}}{\Delta x^{4}}+\alpha_{i} u_{i}=P\left(x_{i}, H\right) \quad i=1,2,3 \cdots N
$$

Then, the continuous problem becomes the following algebraic equation $A U=P$, where

$$
A=\left(\begin{array}{ccccccc}
\frac{7 D}{\Delta x^{4}}+\alpha_{1} & \frac{-4 D}{\Delta x^{4}} & \frac{D}{\Delta x^{4}} & 0 & 0 & \cdots & 0 \\
\frac{-4 D}{\Delta x^{4}} & \frac{6 D}{\Delta x^{4}}+\alpha_{2} & \frac{-4 D}{\Delta x^{4}} & \frac{D}{\Delta x^{4}} & 0 & \ldots & 0 \\
\frac{D}{\Delta x^{4}} & \frac{-4 D}{\Delta x^{4}} & \frac{6 D}{\Delta x^{4}}+\alpha_{3} & \frac{-4}{\Delta x^{4}} & \frac{D}{\Delta x^{4}} & \cdots & 0 \\
\vdots & \ddots & \ddots & \ddots & \ddots & \ddots & \vdots \\
0 & \cdots & \frac{D}{\Delta x^{4}} & \frac{-4 D}{\Delta x^{4}} & \frac{6 D}{\Delta x^{4}}+\alpha_{N-2} & \frac{-4 D}{\Delta x^{4}} & \frac{D}{\Delta x^{4}} \\
0 & \cdots & 0 & \frac{D}{\Delta x^{4}} & \frac{-4 D}{\Delta x^{4}} & \frac{6 D}{\Delta x^{4}}+\alpha_{N-1} & \frac{-4 D}{\Delta x^{4}} \\
0 & \cdots & 0 & \frac{D}{\Delta x^{4}} & \frac{-4 D}{\Delta x^{4}} & \frac{7 D}{\Delta x^{4}}+\alpha_{N}
\end{array}\right)
$$

Proposition 1. Note that $A$ is symmetric positive definite under this assumption $\alpha(x) \geq 0$.

Proof. We will prove that $\alpha(x) \geq 0$ for all $x \in[0, L]$.

For all $(x, y) \in[0, L] \times[0, H]$ we have $\frac{\partial p(x, y)}{\partial y}=f_{2}^{F}(x, y)+\Delta v_{2}(x, y)$, for $y=H$ and $x \in[0, L]$ we have $v_{2}(x, H)=0$, then $\frac{\partial p(x, H)}{\partial y}=f_{2}^{F}(x, H)$. By choosing $f_{2}(x, H)=-g \times \rho^{s} \times h$ [3] where $g$ is the gravity force and $\rho^{s}$ the structure density, we obtain $\frac{\partial p(x, H)}{\partial y} \leq 0$. Finally, we deduce $\alpha(x) \geq 0$ for all $x \in[0, L]$. 


\subsection{Coupled Approximate Problem}

Since $A$ is symmetric positive definite, so we can use the conjugate gradient method to solve the following coupled problem. Find $(v, p) \in\left(H_{0}^{1}\left(\Omega_{F}^{u}\right)\right)^{2} \times L^{2}\left(\Omega_{F}^{u}\right)$ and $U$ so that

$$
\begin{cases}A U=P & \\ \mu \int_{\Omega_{F}^{u}} \nabla v: \nabla w \mathrm{~d} x-\int_{\Omega_{F}^{u}} p \operatorname{div}(v) \mathrm{d} x=\int_{\Omega_{F}^{u}} f^{F} w \mathrm{~d} x, & \forall w \in\left(H_{0}^{1}\left(\Omega_{F}^{u}\right)\right)^{2} \\ \int_{\Omega_{F}^{u}} \operatorname{div}(v) q \mathrm{~d} x=0, & \forall q \in L^{2}\left(\Omega_{F}^{u}\right)\end{cases}
$$

\section{Numerical Method}

To solve numerically the coupled problem we use the following conjugate gradient algorithm.

Proposition 2. Let $A$ be a symmetric positive definite matrix, and $u_{0} \in \mathbb{R}$. Let $\left(u_{k}, r_{k}, z_{k}\right)$ be three sequences defined by the induction relations

$$
z_{0}=r_{0}=P-A u_{0}, \text { and for } k \geq 0\left\{\begin{array}{l}
u_{k+1}=u_{k}+\lambda_{k} z_{k} \\
r_{k+1}=r_{k}-\lambda_{k} A z_{k} \\
z_{k+1}=r_{k+1}+\alpha_{k} z_{k}
\end{array}\right.
$$

with

$$
\alpha_{k}=\frac{\left\|r_{k+1}\right\|^{2}}{\left\|r_{k}\right\|^{2}} \text { and } \lambda_{k}=\frac{\left\|r_{k}\right\|^{2}}{\left(z_{k}, A z_{k}\right)}
$$

Then, $\left(u_{k}\right)_{0 \leq k \leq k_{0}+1}$ is the sequence of approximate solutions of the the conjugate gradient method [7].

\section{Description of the computational method}

Step 1: It computes in the initial field the velocity and the pressure.

Step 2: It uses the conjugate gradient algorithm to find the structure deformation $u$.

Step 3: It computes again the pressure and the velocity in the deformed domain

\section{Numerical Results}

Let the real noted test defined by test $=\|P\|^{2} \times \varepsilon$. We define the stopping criterion of iterations for the conjugate gradient algorithm by rhoold $>$ test and $k<n$ where, $k$ the number of iterations and rhoold $=\left\|z_{0}\right\|^{2}$.

We assume that the velocity on the boundary fluid domain is [3]:

$$
G=\left\{\begin{array}{l}
G_{1}\left(x_{1}, x_{2}\right)=v_{1}=30\left(1-\frac{x_{2}^{2}}{H}\right) \text { and } G_{2}\left(x_{1}, x_{2}\right)=v_{2}=0, \text { on } \Sigma_{1} \\
G_{1}\left(x_{1}, x_{2}\right)=v_{1}=30 \text { on } G_{2}\left(x_{1}, x_{2}\right)=v_{2}=0, \text { on } \Sigma_{2} \\
G_{1}\left(x_{1}, x_{2}\right)=v_{1}=0 \text { and } G_{2}\left(x_{1}, x_{2}\right)=v_{2}=0, \text { on } \Sigma_{3}
\end{array}\right.
$$

We take parameters for fluid and structure in [3] [4]. (Tables 1-3) 
The Table 3 shows that, if we take the tolerance test $=1.95 \times 10^{-3}$ we have the convergence of the algorithm after $k=10$ iterations and rhoold $=5.20 \times 10^{-21}<$ test and the norm of the displacement is $\|u\|_{\infty}=3.3 \times 10^{-3}$.

Freefem $++[8]$ is used for the numerical tests. Figures 2-7 following display the structure displacement, the pressure and the velocity.

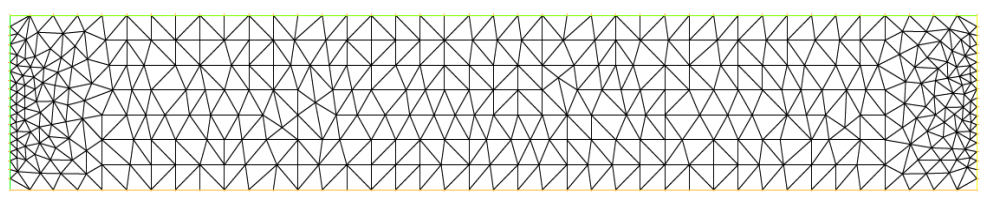

Figure 2. Initial grid.

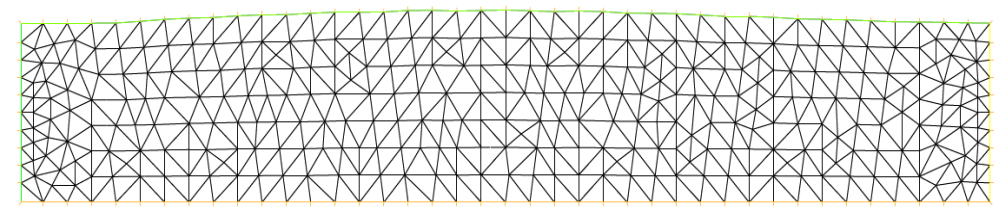

Figure 3. Final grid.

Table 1. Parameters of the strcuture.

\begin{tabular}{cc}
\hline Paramerters & Values \\
\hline$E$ & $0.75 \times 10^{6} \mathrm{~g} / \mathrm{cm}^{2} \cdot \mathrm{s}^{2}$ \\
$h$ & $0.1 \mathrm{~cm}$ \\
$v$ & 0.3 \\
$\rho^{s}$ & $1.1 \frac{\mathrm{g}}{\mathrm{cm}^{3}}$ \\
\hline
\end{tabular}

Table 2. Parameters of the fluid.

\begin{tabular}{cc}
\hline Paramerters & Values \\
\hline$\mu$ & $0.035 \mathrm{~g}$ \\
$H$ & $3 \mathrm{~cm}$ \\
$g$ & $0.5 \mathrm{~cm}$ \\
$f^{F}=\left(f_{1}^{F}, f_{2}^{F}\right)$ & $9.81 \frac{\mathrm{cm}}{\mathrm{s}^{2}}$ \\
\end{tabular}

Table 3. Results related to the algorithm.

\begin{tabular}{cc}
\hline Paramerters & Values \\
\hline$\varepsilon$ & $10^{-6}$ \\
test & $1.95 \times 10^{-3}$ \\
rhoold & $5.20 \times 10^{-21}$ \\
$k$ & 10 \\
$\|u\|_{\infty}$ & $3.3 \times 10^{-3}$ \\
\hline
\end{tabular}


IsoValue
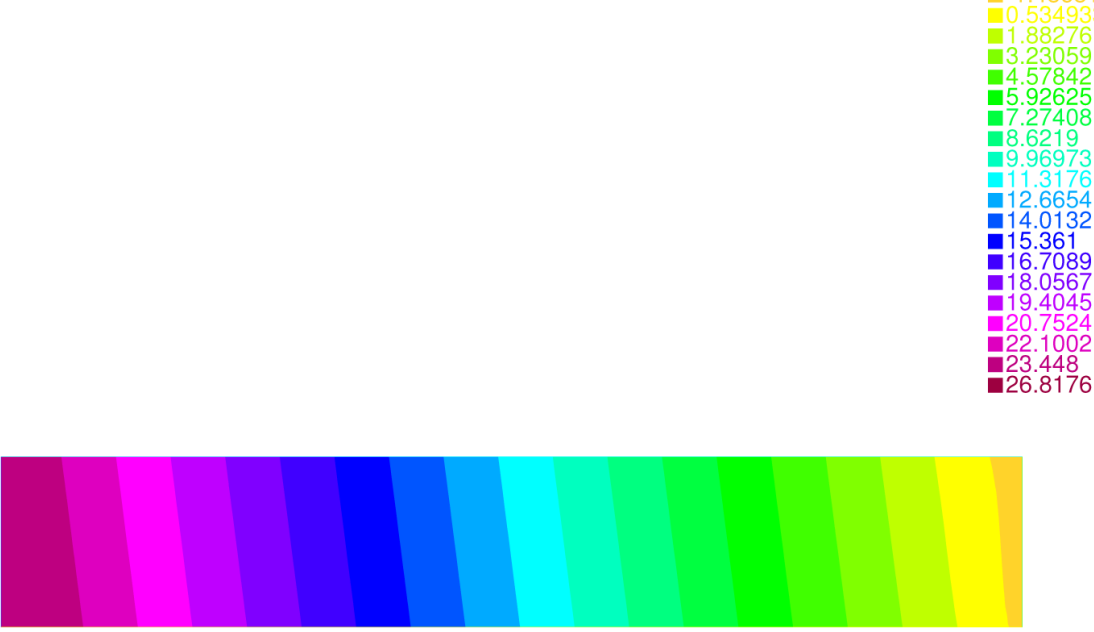

Figure 4. Pressure profile in the initial domain.
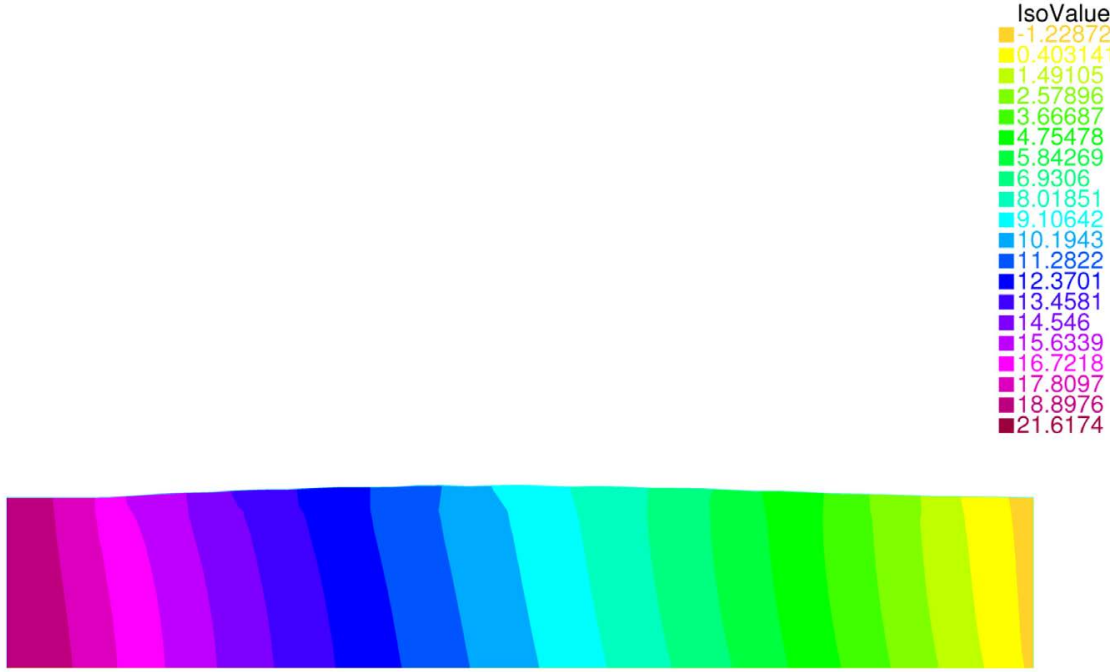

Figure 5. Pressure profile in the deformed domain.
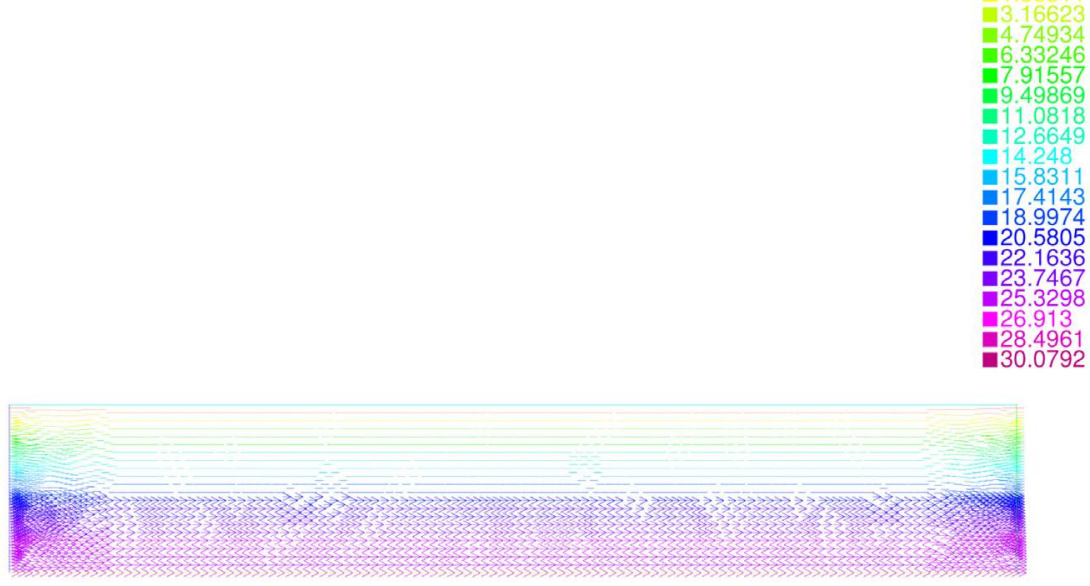

Figure 6. Velocity profile in the initial domain. 


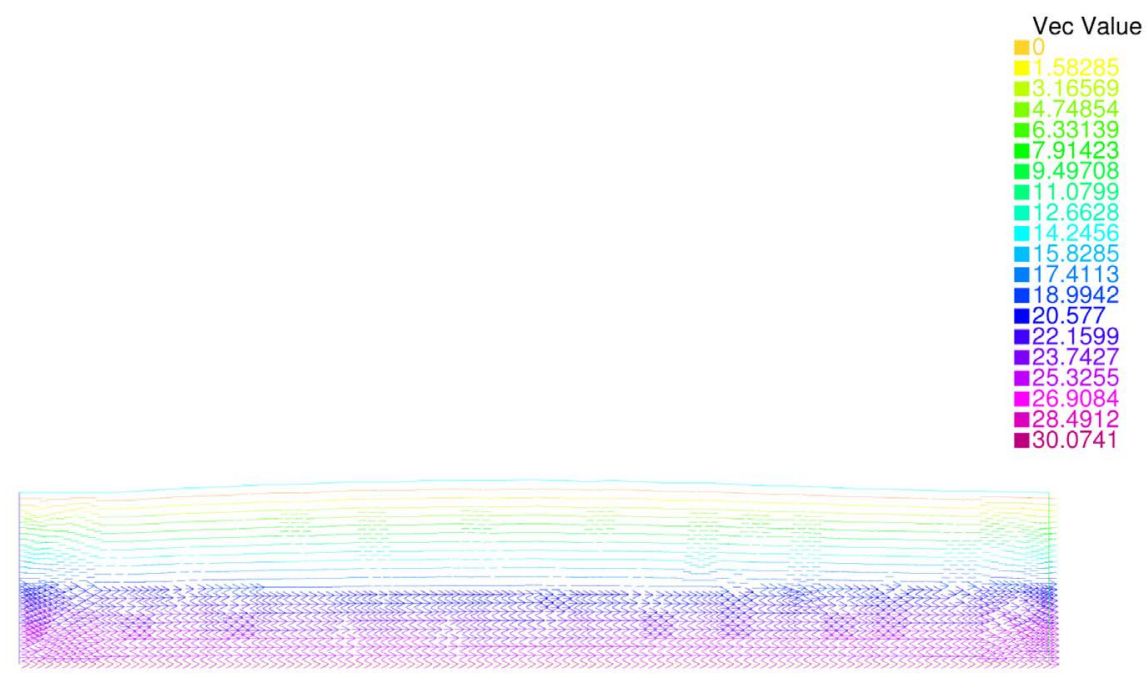

Figure 7. Velocity profile in the deformed domain.

\section{Conclusion}

In this paper, we present a method to solve a steady coupled problem. Our method is based namely on the conjugate gradient algorithm, it takes simultaneously into account three unknown parameters so that each of them depends on the others. To get the results it is necessary to solve the fluid in the initial domain with the finite element method in order to determine the displacement of the structure by the conjugate gradient method and finally to deduce the velocity and the pressure. The velocity, the pressure and the displacement profile compared to [2] [3] [5] appear good. In this work, the only thing to skill is to reduce the number of iterations and to apply this strategy on the unsteady problem.

\section{References}

[1] Mbaye, I. (2012) Fourier Series Development Resulting from an Unsteady Coupled Problem. International Journal of Contemporary Mathematical Sciences, 7, 16951706.

[2] Mbaye, I. (2013) Fourier Series Development for Solving a Steady Fluid Structure Interaction Problem. International Journal of Contemporary Mathematical Sciences, 8, 75-84. https://doi.org/10.12988/ijcms.2013.13008

[3] Murea, C.M. (2005) The BFGS Algorithm for Nonlinear Least Squares Problem Arising from Blood Flow in Arteries. Computers \& Mathematics with Applications, 49, 171-186.

[4] Mbaye, I. and Murea, C. (2007) Numerical Procedure with Analytic Derivative for Unsteady Fluid-Structure Interaction. Communications in Numerical Methods in Engineering, 24, 1257-1275. https://doi.org/10.1002/cnm.1031

[5] Mbaye, I. (2012) Controllability Approach for a Fluid Structure Interaction Problem. Applied Mathematics, 3, 213-216. https://doi.org/10.4236/am.2012.33034

[6] Turek, S. and Hron, J. (2006) Proposal for Numerical Benchmarking of Fluid-Structure Interaction between an Elastic Object and Laminar Incompressible Flow. Computational Science and Engineering, 53, 371-385.

[7] Allaire, G. (2007) Numerical Analysis and Optimization an Introduction to Ma- 
thematical Modelling and Numerical Simulation. Oxford University Press Inc., New York.

[8] Hecht, F. and Pironneau, O. (2003) A Finite Element Software for PDE: FreeFem++. www.freefem.org

Submit or recommend next manuscript to SCIRP and we will provide best service for you:

Accepting pre-submission inquiries through Email, Facebook, LinkedIn, Twitter, etc. A wide selection of journals (inclusive of 9 subjects, more than 200 journals)

Providing 24-hour high-quality service

User-friendly online submission system

Fair and swift peer-review system

Efficient typesetting and proofreading procedure

Display of the result of downloads and visits, as well as the number of cited articles Maximum dissemination of your research work

Submit your manuscript at: http://papersubmission.scirp.org/

Or contact am@scirp.org 